\title{
The role of the clinical research coordinator - data manager - in oncology clinical trials
}

Fernando Rico-Villademoros*1, Teresa Hernando ${ }^{1}$, Juan-Luis Sanz ${ }^{1}$, Antonio López-Alonso ${ }^{1}$, Oscar Salamanca ${ }^{1}$, Carlos Camps ${ }^{2}$ and Rafael Rosell ${ }^{3}$

\author{
Address: ${ }^{1}$ Biometrica, Eloy Gonzalo 27, 28010-Madrid, Spain, ${ }^{2}$ Oncology Department, Hospital General de Valencia, Avda Tres Cruces s/n, 46014 - \\ Valencia, Spain and ${ }^{3}$ Medical Oncology Service, Institut Català d'Oncologia, Hospital Germans Trias i Pujol, Ctra. Canyet s/n, 08916-Badalona \\ (Barcelona), Spain \\ Email: Fernando Rico-Villademoros* - fernando.rico-villademoros@biometrica-group.com; Teresa Hernando - teresa.hernando@biometrica- \\ group.com; Juan-Luis Sanz - juan-luis.sanz@biometrica-group.com; Antonio López-Alonso - antonio.lopez@biometrica-group.com; \\ Oscar Salamanca - oscar.salamanca@biometrica-group.com; Carlos Camps - camps_car@gva.es; Rafael Rosell - rrosell@ns.hugtip.scs.es \\ * Corresponding author
}

Published: 25 March 2004

BMC Medical Research Methodology 2004, 4:6
Received: 23 December 2003

Accepted: 25 March 2004

This article is available from: http://www.biomedcentral.com/I47I-2288/4/6

(C) 2004 Rico-Villademoros et al; licensee BioMed Central Ltd. This is an Open Access article: verbatim copying and redistribution of this article are permitted in all media for any purpose, provided this notice is preserved along with the article's original URL.

\begin{abstract}
Background: The purpose of this study was to determine the standard tasks performed by clinical research coordinators (CRCs) in oncology clinical trials.

Methods: Forty-one CRCs were anonymously surveyed, using a four-page self-administered questionnaire focused on demographics, qualifications, and professional experience. The survey questions on responsibilities consisted of an ad-hoc 32-item questionnaire where respondents had to rate the frequency of involvement in the listed activities using a 3-point scale. We defined as "standard" a task that was rated as "in all or nearly all trials" by at least half of the respondents.
\end{abstract}

Results: A response rate of $90 \%$ (37 out of $4 \mathrm{I}$ ) was achieved after two mailings. Less than half of the respondents had received additional training in oncology, clinical research or Good Clinical Practices (GCP). Overall, all standard tasks performed by CRCs were in the category of "monitoring activities" (those usually performed by a Clinical Research Associate "CRA") and included patient registration/randomization, recruitment follow-up, case report form completion, collaboration with the CRA, serious adverse events reporting, handling of investigator files, and preparing the site for and/or attending audits.

Conclusions: CRCs play a key role in the implementation of oncology clinical trials, which goes far beyond mere data collection and/or administrative support, and directly contributes to the gathering of good quality data.

\section{Background}

Randomized, controlled trials represent the most definitive method to determine the effectiveness or ineffectiveness of a cancer intervention $[1,2]$. In addition, when meeting very rigid medical, scientific and ethical standards, they also represent "Good Medicine" (i.e. a standard of care) [2]. 
Nowadays it is widely accepted that the design, implementation, coordination and analysis of modern clinical trials require a multidisciplinary specialist approach [3]. The basic research team includes the principal investigator, sub-investigators, clinical monitors (also called clinical research associates or "CRAs"), the data management team, statisticians, and a relatively recent position: the clinical research coordinator (CRC). The CRC, regardless of job title (study or site coordinator, data manager, research nurse, etc), plays a key role in the clinical trial process [3-8].

The responsibilities of investigators, sponsors and monitors are well defined in the Spanish regulation on clinical trials [9], FDA regulations [10] and international guidelines on Good Clinical Practice [11]. Despite the abovementioned importance of the CRC position however, their responsibilities and main tasks have not been formally described. Furthermore, literature on this topic is limited since most papers mainly address the required skills of a CRC $[4,8,12]$, or describe their responsibilities within a specific project $[13,14]$. The British Oncology Data Managers Association (BODMA) has run several surveys among its members $[3,15]$ but very little data has been published in journals accessible by commonly used literature databases (e.g. MEDLINE) [3]. Raybuck [16], in a review of the literature, concluded that their role is vague and ill defined, with little emphasis on the need for advanced educational preparation. Arrigo et al [7] carried out the only published survey that we are aware of addressing this issue. They surveyed 120 nurses in the EORTC Oncology Nurses Study Group. Their results indicate that nurses' tasks and activities are oriented primarily toward patient care, such as informing patients, drug preparation and administration, monitoring of toxicities, organization of follow-up, basic patient care, and data management. However, we think that this otherwise interesting survey has two important limitations. First of all, only nurses were surveyed, and it is well recognized that CRCs have a broader range of backgrounds $[15,17]$. Secondly, their report did not state the content and format of the questions used, and therefore we cannot know whether these activities are the standard ones (i.e. activities which are performed by most of the respondents in most of the trials).

The purpose of our study was to determine the standard tasks performed by CRCs in oncology clinical trials. In addition, we assessed their job satisfaction and training needs.

\section{Methods}

\section{Survey creation}

We created a four-page self-administered questionnaire with three main sections:

\section{Demographic data: sex and age}

2. Qualifications: highest educational level of respondents, language skills (upper-intermediate level), and other training received. The latter was included to ascertain whether they received any structured training in oncology, clinical research, clinical trials, statistics, Good Clinical Practice, data management, or other training.

3. Professional experience: this was the core section of the survey. We collected data on: current and previous experience as a CRC; previous experience in the pharmaceutical industry or in a contract research organization (CRO); current job title; workload, defined as the number and phases of the clinical trials they were currently participating in (i.e. those currently recruiting patients), number of patients included and number of monitoring visits received per month; responsibilities (see below); job importance, determined using a visual analogue scale of 1 ("not important") to 10 ("very important"); job satisfaction, using a 7-item likert scale where 1 was "extremely dissatisfied", 4 "neither satisfied nor dissatisfied", and 7 "extremely satisfied"; and finally an evaluation of training needs.

The survey questions on responsibilities were created adhoc since we did not find any such questionnaire in the literature. In the first draft, we listed tasks related to the whole clinical trial process with which investigators, CRAs and ancillary personnel are usually involved. We added two general data management items, and one statistician question. We interviewed 2 CRCs by phone and asked them to list all the activities they usually perform. Any activities that were not originally in the first version were included. All authors reviewed this second version and a final 32-item questionnaire was agreed upon (Figure 1). Respondents were asked to rate the frequency of involvement in the listed activities using a 3-point scale, modified from a format developed by the RAND Corporation for ascertaining expert consensus [18]. In this scale, 1 was "in none or few trials" ( $\leq 25 \%$ of the trials they are currently participating in), 2 was "in some trials" (26-75\% of the trials), and 3 was "in all or nearly all trials" ( $>75 \%$ of the trials). A copy of this questionnaire and the complete survey are available from the authors upon request.

\section{Sampling method}

The Spanish Lung Cancer Group sent this anonymous survey to 41 CRCs who were going to attend a course on clinical trials in oncology. An accompanying letter explained the aim of the survey and indicated the deadline for returning it either by fax or ordinary mail. A reminder was sent by the study co-sponsor (Biométrica) to solicit outstanding questionnaires. 
Administrative activities

Management of IRB submission

Management of Hospital Director submission

Management Medicine Agency submission

Medical history adaptation for the CT

Scheduled protocol specified tests

Scheduled patient's CT appointments

\section{Clinical activities}

To identify potential eligible patients

Assessment of inclusion/exclusion criteria

Participation in informing of patients

Participation in obtaining informed consent

To assess response to therapy

To assess toxicities

Scales/questionnaires completion

\section{Monitoring activities}

Patient registration/randomization

To deal with Hospital Pharmacy

To deal with a central lab

Recruitment follow-up

CRF completion

To act as CRA

To collaborate with the CRA

Queries resolution

Reporting serious adverse events

To handle the investigator file

To prepare and/or attend audits

\section{Data management and statistics \\ Database set-up \\ Data entry \\ Statistical analysis}

\section{Researcher-related activities}

Participation in protocol/CRF design

Participation in protocol/CRF review

Attending investigators meeting

Participation in Final Report

Participation in CT publication
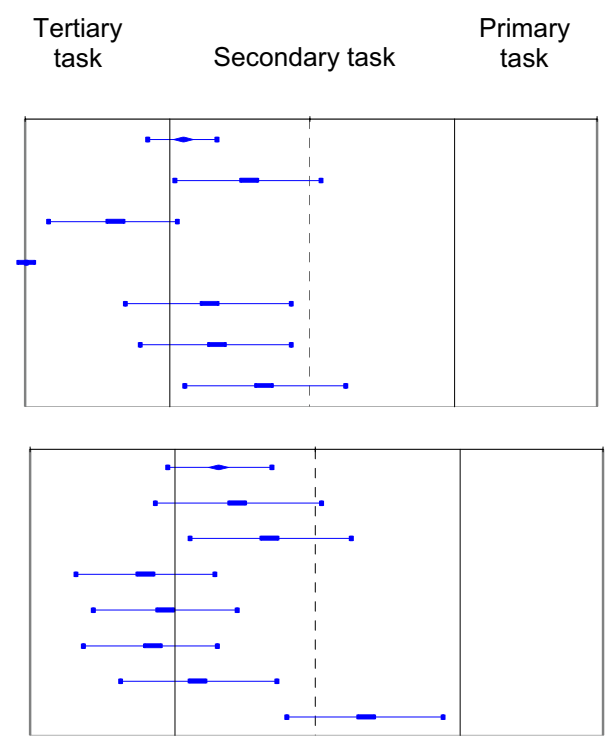

Mean (SD) Scoring '3'

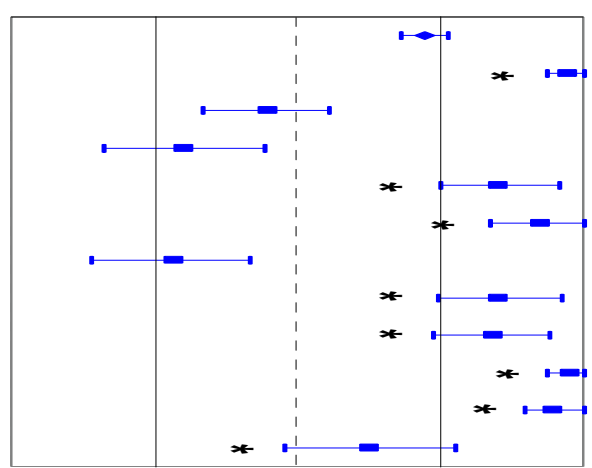

\begin{tabular}{c|c}
\hline $\mathbf{1 . 5 5}(\mathbf{0 . 3 6 )}$ & $\boldsymbol{N} . \boldsymbol{A}$. \\
\hline $1.78(0.76)$ & $19.4 \%$ \\
\hline $1.31(0.67)$ & $11.1 \%$ \\
\hline $1.00(0.00)$ & $0.0 \%$ \\
\hline $1.64(0.86)$ & $25.0 \%$ \\
\hline $1.67(0.79)$ & $19.4 \%$ \\
\hline $\mathbf{1 . 8 3}(\mathbf{0 . 8 4})$ & $27.8 \%$ \\
\hline \multicolumn{2}{|c}{} \\
\hline $\mathbf{1 . 6 6}(\mathbf{0 . 5 4})$ & $\boldsymbol{N} . \boldsymbol{A}$. \\
\hline $1.72(0.85)$ & $25.0 \%$ \\
\hline $1.83(0.85)$ & $27.8 \%$ \\
\hline $1.40(0.70)$ & $11.4 \%$ \\
\hline $1.47(0.74)$ & $13.9 \%$ \\
\hline $1.42(0.69)$ & $11.1 \%$ \\
\hline $1.58(0.81)$ & $19.4 \%$ \\
\hline $\mathbf{2 . 1 7}(\mathbf{0 . 8 1})$ & $41.7 \%$ \\
\hline
\end{tabular}

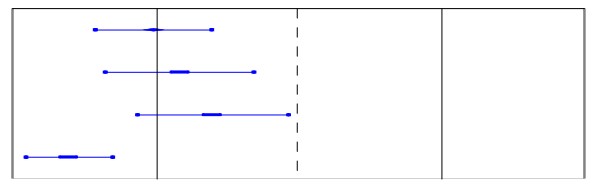

\begin{tabular}{c|c}
\hline $\mathbf{1 . 4 9}(\mathbf{0 . 5 9 )}$ & $\boldsymbol{N} . \boldsymbol{A}$. \\
\hline $1.58(0.77)$ & $16.7 \%$ \\
\hline $1.69(0.79)$ & $19.4 \%$ \\
\hline $\mathbf{1 . 1 9}(\mathbf{0 . 4 7 )}$ & $2.8 \%$ \\
\hline
\end{tabular}

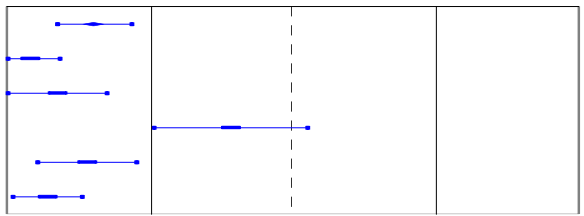

\begin{tabular}{c|c}
\hline $\mathbf{1 . 3 0}(\mathbf{0 . 3 8 )}$ & $\boldsymbol{N} . \boldsymbol{A}$. \\
\hline $1.08(0.28)$ & $0.0 \%$ \\
\hline $1.17(0.51)$ & $5.7 \%$ \\
\hline $1.78(0.80)$ & $22.2 \%$ \\
\hline $1.28(0.51)$ & $2.8 \%$ \\
\hline $\mathbf{1 . 1 4}(\mathbf{0 . 4 0 )}$ & $0.0 \%$ \\
\hline
\end{tabular}

1

\begin{tabular}{c|c}
\hline $\mathbf{2 . 4 5}(\mathbf{0 . 2 7})$ & $\boldsymbol{N} . \boldsymbol{A}$. \\
\hline $2.94(0.23)$ & $94.4 \%$ \\
\hline $1.89(0.66)$ & $16.2 \%$ \\
\hline $1.60(0.81)$ & $20.0 \%$ \\
\hline $2.70(0.62)$ & $78.4 \%$ \\
\hline $2.84(0.50)$ & $89.2 \%$ \\
\hline $1.56(0.81)$ & $19.4 \%$ \\
\hline $2.70(0.54)$ & $74.1 \%$ \\
\hline $2.68(0.63)$ & $75.7 \%$ \\
\hline $2.95(0.23)$ & $94.6 \%$ \\
\hline $2.89(0.32)$ & $89.2 \%$ \\
\hline $\mathbf{2 . 2 5 ( 0 . 8 7 )}$ & $52.8 \%$ \\
\hline
\end{tabular}

\section{* Standard Tasks (i.e. those performed by at least $50 \%$ of the respondents in most of the trials)} 1 :"in none or few trials"; $2=$ "in some trials"; $3=$ "in all or near all trials" Abbreviations: CRA: clinical research associate; CRF: case report form; IRB: institutional review board

\section{Figure I}

Tasks of the Clinical Research Coordinators 


\section{Data analysis}

Returned surveys were checked for duplication using three variables: age, sex, and geographical region where the CRC was working. Only the first reply was tabulated. After study data were tabulated and quality control was performed, the results were analyzed using the statistical package SPSS, version 10.0 [19].

In the descriptive analysis, the mean or median, standard deviation and range were calculated for the quantitative variables, and the frequency and percentage of answers in each category for the qualitative variables. Answers to the questionnaire on responsibilities were analyzed in the following manner. We first calculated the mean, standard deviation and 95\% confidence interval (CI) for each item. We defined as "standard" any CRC task that was rated as a 3 ("in all or near all trials") by at least half of the respondents. In addition, CRC activities were grouped somewhat arbitrarily into five categories: administrative (those that could be done by a trained secretary); clinical (those which required direct contact with the patient and/ or required some degree of clinical training/background); monitoring (those usually performed by a CRA); data management and statistics (those usually performed by the data management department [i.e. database set-up and data entry] and the statistics department [i.e. statistical analysis]); and researcher-related activities (those commonly performed by investigators).

Finally, an exploratory univariate analysis was performed to test whether background (medical/nursing vs. others) or frequency of participation in multinational clinical trials influenced the job profile. Pairwise comparisons were performed using the Mann-Whitney test, and they were considered significant if $P \leq 0.05$ (two-sided test).

\section{Results}

A response rate of $90 \%$ (37 out of 41) was achieved after two mailings. Most of the respondents were female (89.2\%). Mean age was 30.4 years, ranging from 22 to 51 . The majority of respondents $(89.2 \%)$ held a university degree and had an English fluency level they considered upper-intermediate $(\mathrm{n}=34,91.9 \%)$. Qualifications are detailed in Table 1.

\section{Professional experience and workload}

More than two-thirds of the respondents ( $\mathrm{n}=26,74.3 \%)$ were called "Data Managers" while the title "Clinical Research Coordinator" (i.e. titles that included both terms "research" and "coordinator") appeared in only 3 (8.6\%) job titles. Mean duration of coordinator experience in the current position and as a whole were 27.6 (SD: 29.7) and 36.9 (SD: 43.2) months respectively. Two-thirds of the CRCs (23 out of 35 respondents, 65.7\%) were devoted full-time to their position, and only $24.3 \%(n=9)$ had previous experience in a similar position. Previous experience in the pharmaceutical industry or a CRO was reported by $8.1 \%$ and $5.4 \%$ of the respondents, respectively. Data on the CRCs' workload, expressed by the number of trials they were currently involved in, type of trial, and number of monitoring visits received, is presented in Table 2.

Table I: Qualifications of the respondents

\begin{tabular}{|c|c|c|}
\hline Educational level/Training received & No. of CRCs & $\%$ \\
\hline University degree & 31 & 83.8 \\
\hline Medicine & 8 & 21.6 \\
\hline Pharmacy & 5 & 13.5 \\
\hline Biology & 5 & 13.5 \\
\hline Nursing & 6 & 16.2 \\
\hline Other & 7 & 19.0 \\
\hline \multicolumn{3}{|l|}{ Training received } \\
\hline Oncology & 12 & 32.4 \\
\hline Clinical Research & 17 & 45.9 \\
\hline Clinical Trials & 20 & 54.1 \\
\hline GCP* & 15 & 40.5 \\
\hline Statistics & 8 & 21.6 \\
\hline Database preparation \& management & 9 & 24.3 \\
\hline
\end{tabular}

*GCP: Good clinical Practice 
Table 2: Workload of the Clinical Research Coordinators

\begin{tabular}{|c|c|c|c|}
\hline Parameter & $\mathbf{N}$ & Median & Range \\
\hline No. of current CTs* & 37 & 12.5 & $2.0-46.0$ \\
\hline No. of multinational CTs & 33 & 4.0 & $0.0-17.5$ \\
\hline No. of CTs by sponsorship: & 34 & & \\
\hline Pharmaceutical Industry & & 4.0 & $0.0-30.0$ \\
\hline Cooperative Groups & & 8.0 & $0.0-22.0$ \\
\hline No. of CTs by Phase: & 30 & & \\
\hline Phase I & & 0.0 & $0.0-3.0$ \\
\hline Phase II & & 5.0 & $0.0-14.0$ \\
\hline Phase III & & 5.0 & $0.0-28.0$ \\
\hline Phase IV & & 0.5 & $0.0-5.0$ \\
\hline Compassionate & & 0.0 & $0.0-3.0$ \\
\hline No. of CTs by Indication: & 27 & & \\
\hline Breast cancer & & 3.0 & $0.0-21.0$ \\
\hline Lung cancer & & 3.0 & $0.0-13.0$ \\
\hline Colorectal cancer & & 2.0 & $0.0-9.0$ \\
\hline Head \& Neck cancer & & 0.0 & $0.0-4.0$ \\
\hline Ovarian cancer & & 0.0 & $0.0-2.0$ \\
\hline Other & & 0.0 & $0.0-8.0$ \\
\hline No. of patients: & 33 & & $\%$ \\
\hline $0-25$ & & NA & 30.3 \\
\hline $26-50$ & & NA & 15.2 \\
\hline $5 I-75$ & & NA & 6.1 \\
\hline $76-100$ & & NA & 12.1 \\
\hline$>100$ & & NA & 36.3 \\
\hline $\begin{array}{l}\text { No. of monitoring visits received: } \\
\text { per month }\end{array}$ & & 2.0 & $0.0-10.0$ \\
\hline
\end{tabular}

*Current CTs: those that were currently recruiting patients CTs: clinical trials; N: number of respondents; NA: not applicable

\section{Clinical trial coordinator's tasks}

The responsibilities questionnaire produced a list of CRC tasks, identified in Figure 1. In analyzing the responses to these survey questions, we first calculated the mean, standard deviation and $95 \%$ CI for each item. The CI for each task is represented by a horizontal bar, and the numerical values are given in the table on the right. The table also shows the percentage of respondents who rated the task as a 3. The CI chart can be interpreted in two complementary ways. Standard tasks (those having been rated as a "3" by at least half of the respondents) are indicated with an asterisk in the graphic. In addition, we have divided the chart into three columns representing primary, secondary and tertiary tasks. When the confidence interval falls entirely within the first column, we have considered it a "primary task" - one performed by most respondents in most trials. Similarly, "secondary tasks" are those performed by some or many of the respondents in most trials, and "tertiary tasks" those performed by few of the respondents in most trials.

Overall, CRCs seem to be devoted to what we have called "monitoring activities" (Figure 1). All standard tasks fell into this category, including patient registration/randomization, recruitment follow-up, CRF completion, collaboration with the CRA, Serious Adverse Events (SAE) reporting, investigator file handling, and preparing the site for and/or attending audits. In addition, it was not uncommon for the CRC to deal with the Hospital Pharmacy. To a lesser extent, they were also involved in some administrative activities (such as IRB submission and scheduling patient's CT appointments), and various clinical activities (inclusion/exclusion criteria assessment and completion of scales/questionnaires, ). Data management and researcher-related activities as defined above were underrepresented among the CRCs surveyed. 


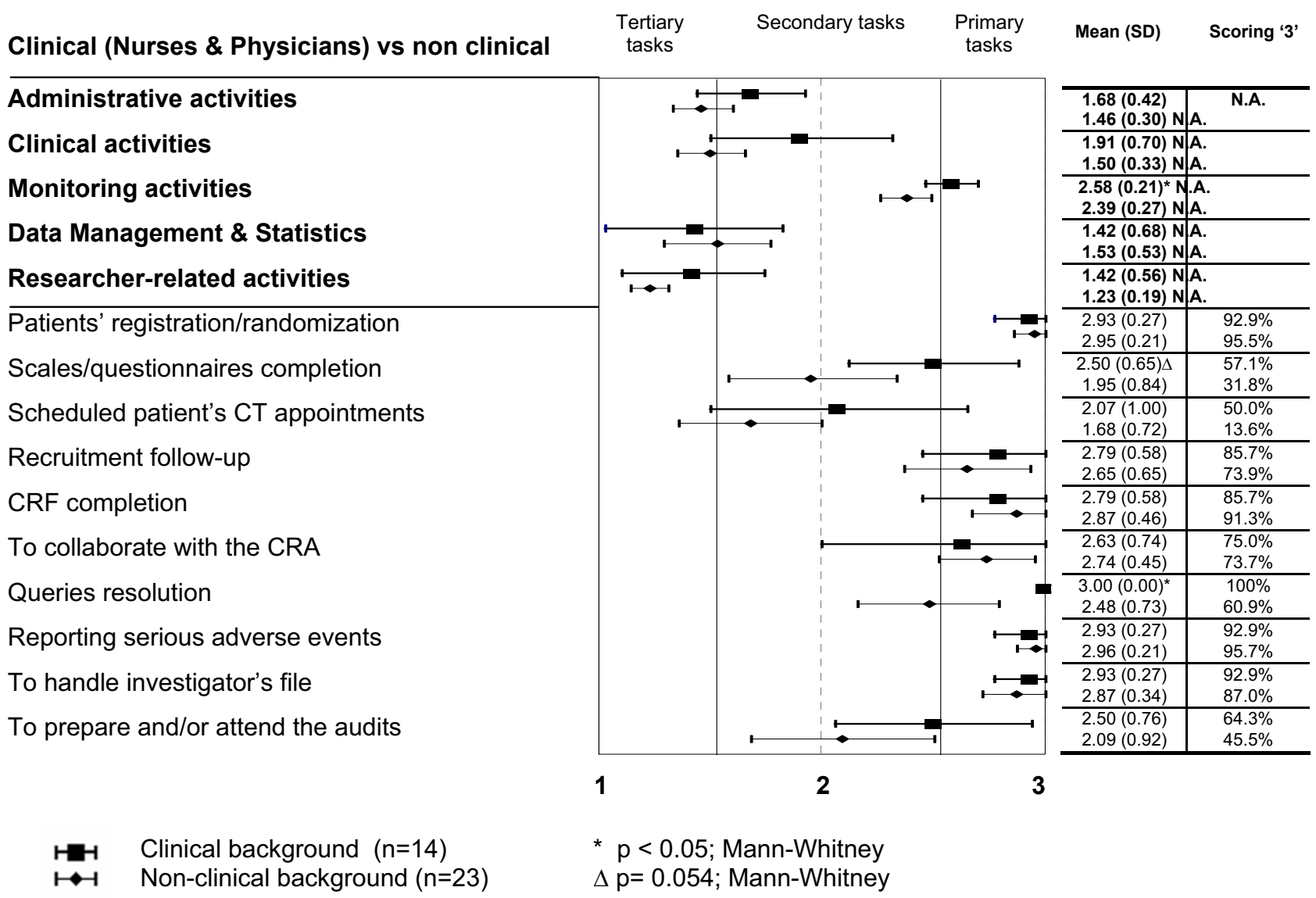

Figure 2

CRC's Tasks according to their educational background

Figures 2 and 3 show tasks profiles, and consider background (physicians and nurses vs. non-clinical) and degree of involvement in multinational clinical trials. For the latter, the group was divided into those involved in $\leq$ 4 multinational trials, and those involved in $>4$. We present data for the category headings as well as for those items that turned out to be standard tasks in either group. This analysis suggests that the overall task profile is the same regardless of the CRC's background or their involvement in multinational trials. In any case, the CRCs are mainly devoted to monitoring activities.

However, some interesting differences were found. Nurses and physicians had greater involvement in clinical activities, such as completion of scales/questionnaires $(P=$ $0.054)$ and, although they were not rated as standard tasks, response to therapy assessment $(1.86 \pm 0.86$ vs. 1.14 $\pm 0.36, P<0.05)$ and toxicity assessment $(2.14 \pm 0.86$ vs. $1.24 \pm 0.54, P<0.05)$. In addition, query resolution was performed "in all or near all trials" by $100 \%$ of the nurses/ physicians, compared to $60.9 \%$ of the CRCs with a different background. CRCs with greater involvement in multinational clinical trials were also more involved in CRF completion, query resolution and audits (Figure 3 ).

\section{Job importance and satisfaction}

Most of the surveyed CRCs believed they play an important role in oncology clinical trials (mean VAS score 7.6, $\mathrm{SD}=1.2, \mathrm{~N}=37$ ). Eighty-three percent of the respondents were either extremely or very satisfied with their job.

\section{Discussion}

Our study has several limitations. First of all, our sample size could be considered small although it represents 30\% (37 out of 120) of the CRCs currently working with the Spanish Lung Cancer Group. Secondly, a selection bias exists since we only surveyed those CRCs attending a course on clinical trials. This might explain the high 


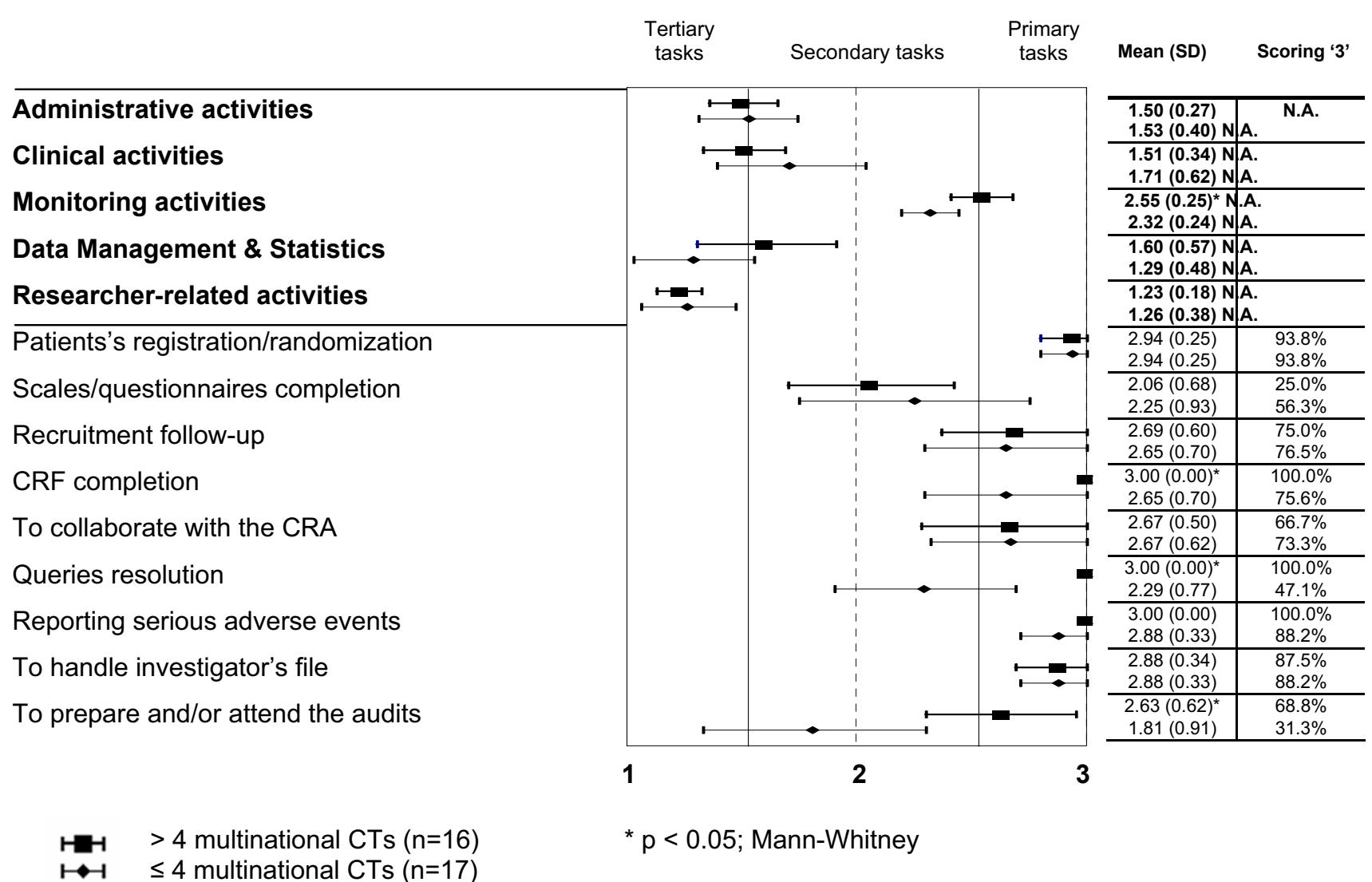

Figure 3

CRC's Tasks according to the involvement in multinational CTs

response rate to the survey. However, it is doubtful that this bias affected the core results (i.e. identifying CRCs tasks), since CRC's mean overall experience was 3 years and we assume they have had enough experience to illustrate the standard tasks of a CRC. However, this bias might have affected secondary variables we studied. As the CRCs interviewed were all attending training, they could be considered highly motivated in their work, which might bias both their job satisfaction as well as the importance they attribute to this role. Finally, we cannot be assured that the task profile described is applicable to other countries, as there lacks a systematic evaluation of this topic.

In keeping with the findings of other authors, [15,17] our sample contained a wide range of backgrounds. Although most of the respondents held a university degree in health sciences, it seems there are important training needs which need to be addressed in areas such as oncology, clinical trials, and especially GCP. This situation has also been described in other reviews or studies carried out in
Europe $[3,7,15]$. Well-trained and experienced CRCs can contribute significantly to the quality of data collected [20]. At a minimum, we recommend an induction course for those professionals recently appointed as CRCs. We encourage those with greater working experience to obtain certification, such as that provided by the Association of Clinical Research Professionals [21]. Most importantly, GCP training is a key issue when conducting clinical trials, and should be standard for those in charge of data collection and its integrity, such as the CRCs surveyed in our study. To highlight this point, the main objectives of the recently formed EORTC CRC Group are to make a positive impact upon the quality of clinical trials, and create conditions and standards for implementing and conducting clinical protocols according to Good Clinical Practice [22].

Clinical research coordinators' main tasks seem to be monitoring activities, regardless of their background. Not unexpectedly, in addition to monitoring activities, nurses and physicians performed more clinical activities than 
those who did not have a clinical background. Some authors have emphasized that nursing is the ideal discipline from which to assume the role of CRC $[5,16]$. Our results do not support this premise: nurses and physicians who were appointed as CRCs took care of monitoring activities more often than clinical ones. We suggest that CRCs with a clinical background, in addition to the responsibilities associated with that role, are well suited to take on some investigators' responsibilities and thus act also as sub-investigators. The possibility of assuming this double role can be an advantage, but it is important to keep in mind their key tasks as CRCs: to ensure that clinical trials run smoothly and preserving data quality. This situation has been well described by Ocker et al [23] who pointed out that while the main focus of the staff nurses is to provide patient care, the main focus of the research nurse's role is the implementation of the protocol. In fact, the two major advantages described in the literature of having a CRC involved in clinical trials are: (a) to increase recruitment rate $[14,24]$ and (b) to have good data quality [20]. Interestingly, the profile we discovered fits quite well with a definition provided by the Association of Clinical Research Professionals [25]: "A Clinical Research coodinator, Study Site Research Nurse or Study Site Coordinator, works at a clinical research site under the immediate direction of a principal investigator, whose research activities are conducted under Good Clinical Practice regulations. Among other tasks, CRC's peform site preparation, patient screening and recruitment, patient enrolment, conduct and ensure the quality of case report forms, maintain source documents, and ensure site quality".

We found differences between CRCs with greater involvement in multinational clinical trials, and those with less involvement. This may be explained by the fact that multinational clinical trials are more frequently sponsored by the pharmaceutical industry, which imposes higher standards of quality. This may also explain why CRCs involved in multinational trials play a greater role in query resolution and audit.

As data management activities are under-represented amongst the tasks usually performed by CRCs, we advocate the use of "Clinical Research Coordinator" as the job title instead of "Data Manager". Alternatively, both terms could be maintained: (a) data managers for those dealing with data management activities (e.g. database set-up and data entry) and who are usually working at a research center; and (b) CRC for those involved in on-site activities, such as those identified in our study as standard tasks. We also suggest that principal investigators rethink the degree of involvement their CRCs have in certain research activities, as these are also under-represented within standard tasks, as we have defined them. Key personnel involved in the clinical trial process should be familiar- ized with study procedures; therefore CRCs should attend investigators meetings more frequently. Participation in protocol/CRF review and design should also be increased, since the CRC can provide a worthy perspective on the logistics of proposed study procedures [12]. In addition, the involvement of CRCs in such activities will have a positive influence on their job satisfaction [26].

Workloads seem to vary greatly amongst the CRCs surveyed, measured by the number of clinical trials they are coordinating and the number of patients included. Those with greater workloads were more often full time employees, as opposed to those with a lesser workload ( $82.4 \%$ vs. $50 \%, \mathrm{p}=0.07)$. Finally, CRCs in our study appeared to be highly satisfied with their jobs, a finding similar to that observed by Kellen et al [26].

\section{Conclusion}

Clinical Research Coordinators play a key role in the implementation of oncology clinical trials, extending far beyond mere data collection and/or administrative support, and directly contribute to the gathering of good quality data. In line with these responsibilities, investigators and sponsors should take responsibility to ensure that CRCs have adequate training in areas that we have highlighted.

Further research is needed to systematically evaluate the role of the CRC in other therapeutic areas, and in other countries. In addition, it would be valuable to run a similar survey among investigators, in order to ascertain whether the standard tasks CRCs are performing are in line with those expected of this position.

\section{Competing interests}

None declared.

\section{Authors' contributions}

FR initiated and designed the project, prepared the statistical analysis plan and wrote the initial draft of the manuscript. TH interviewed two Clinical Research Coordinators to prepare the final case report form and took care of the survey logistics. JLS prepared the statistical analysis plan and analyzed the data. TH, JLS, AL and OS critically revised the case report form. CC and RR reviewed the case report form and supervised study performance within the Spanish Lung Cancer Group. All authors reviewed the manuscript and approved the final draft.

\section{Acknowledgments}

We wish to thank Elizabeth Breedlove for her assistance with the manuscript, José Javier Sánchez for his critical review of the statistical section, and María Fernández for assisting with the survey. 


\section{References}

I. Benson B III, Prokop J, Bean JA, Rademaker AW, Eshler B, Anderson $\mathrm{K}$ : Oncologists' reluctance to accrue patients onto clinical trial: an Illinois Cancer Center study. J Clin Oncol 1991, 9:2067-2075.

2. Kaufman D: Cancer therapy and the randomized clinical trial: good medicine? CA 1994, 44:109-II4.

3. Riley D, Ward L, Young T, for the British Oncology Data Managers Association (BODMA): Oncology data management in the UK - BODMA's view. Br J Cancer 1994, 70:391-394.

4. Warlow C: Organise a multicentre trial. BMJ I990, 300: I80-I83.

5. Cassidy J, Macfarlane DK: The role of the nurse in clinical cancer research. Cancer Nurs 1991, 14:|24-131.

6. The DCCT Research Group: The impact of the trial coordinator in the Diabetes Control and Complications Trial (DCCT). Diabetes Educ 1993, 19:509-512.

7. Arrigo C, Gall H, Delogne A, Molin C: The involvement of nurses in clinical trials. Results of the EORTC Oncology Nurses Study Group survey. Cancer Nurs 1994, I 7:429-433.

8. Brown JM, Haining SA, Hale JM: Views on local data management in cancer clinical trials. Clin Oncol (R Coll Radlol) 1997, 9:403-406.

9. REAL DECRETO 56I/I993 Establishing requirements concerning clinical trials on drugs. Spain . 16 April 1993

10. Department of Health and Human Services: Investigational New Drug Application. Responsibilities of Sponsors and Investigators, 21 Code of Federal Regulations $\$ 3312.50-312.70$. Revised as of April I, 2003

I I. The European Agency for the Evaluation of Medicinal Products, ICH Topic E 6. Guideline for Good Clinical Practice. Note for Guidance on Good Clinical Practice (CPMP/ICH/ 135/95).

12. Pelke S, Easa D: The role of the clinical research coordinator in multicenter clinical trials. JOGNN 1997, 26:279-285.

13. Mullin SM, Warwick S, Akers M, Beecher P, Helminger K, Moses B, Rigby PA, Taplin NE, Werner W, Wettach R, and the MILIS Study Group: An acuteintervention trial: the research nurse coordinator's role. Control Clin Trials 1983, 5:|4|-I56.

14. O'Halloran LJ, Curl VR, Hagen L, Sveningson L: A research nurse: enhancing participation in clinical trials. Prog Clin Biol Res 1989, 293:355-360.

15. Ablett S: BODMA History. [http://www.bodma.com/bodma.asp] October, 1999. Accessed April 18th, 2004.

16. Raybuck J: The clinical nurse specialist as research coordinator in clinical drug trials. Clin Nurse Spec 1997, I I: I5- 19.

17. Stephens L, Papke L: Certified clinical research coordinators. Appl Clin Trials 1995, 4:58-63.

18. Brook RH, Chassin MR, Fink A, Solomon DH, Kosecoff J, Park RE: A method for the detailed assessment of the appropriateness of medical technologies. Int J Technol Assess Health Care 1986, 2:53-63.

19. SPSS for Windows software application. Version 10.0. Chicago, IL: SPSS Inc 1999.

20. Vantongelen K, Rotmensz N, van der Schueren E, for the EORTC Study Group on Data Management: Quality Control of Validity of Data Collected in Clinical Trials. Eur J Cancer Clin Oncol 1989, 25: | $24 \mid-1247$.

21. ACRP European Certification [http://www.acrpnet.org/certifica tion/ich/index.html]. Accessed April I8th, 2004

22. Fishwick K, Berridge J, Coffey M, Colussi AM, Di Giulio P, Marinus A, Molin C, Peters M: The EORTC Clinical Research Coordinators Group. Eur J Cancer 2002, 38:S54-S59.

23. Ocker B, Plank DM: The research nurse role in a clinic-based oncology research setting. Cancer Nurs 2000, 23:286-289.

24. Isaacman DJ, Reynolds EA: Effect of a research nurse on patient enrolment in a clinical study. Pediatr Emerg Care 1996, I 2:340-342.

25. CCRC Exam Information [http://www.acrpnet.org/education/ examreu/index.html]. Accessed April I8th, 2004

26. Kellen JC, Schron EB, McBride R, Hale C, Campion J, Handshaw K, Inkster $M$, Ettinger A: A survey of clinical trial coordinators: factors influencing job satisfaction and turnover. Cardiovasc Nurs |994, 30:25-3|.

\section{Pre-publication history}

The pre-publication history for this paper can be accessed here:

http://www.biomedcentral.com/1471-2288/4/6/prepub
Publish with Biomed Central and every scientist can read your work free of charge

"BioMed Central will be the most significant development for disseminating the results of biomedical research in our lifetime. "

Sir Paul Nurse, Cancer Research UK

Your research papers will be:

- available free of charge to the entire biomedical community

- peer reviewed and published immediately upon acceptance

- cited in PubMed and archived on PubMed Central

- yours - you keep the copyright

Submit your manuscript here:

http://www.biomedcentral.com/info/publishing_adv.asp
BioMedcentral 